\title{
WEIGHT-PARTIALLY GREEDY BASES AND WEIGHT-PROPERTY
}

\author{
DIVYA KHURANA
}

\begin{abstract}
In this paper, motivated by the notion of $w$-Property $(A)$ defined in [2, we introduce the notions of $w$-left Property $(A)$ and $w$-right Property $(A)$. We also introduce the notions of $w$-partially greedy basis (using a characterization of partially greedy basis from 4) and $w$-reverse partially greedy basis. The main aim of this paper is to study (i) some characterizations of $w$-partially greedy and $w$-reverse partially greedy basis $(i i)$ conditions on the weight sequences when $w$-left Property $(A)$ and (or) $w$-right Property $(A)$ implies $w$-Property $(A)$.
\end{abstract}

\section{INTRODUCTION}

Let $X$ be a real Banach space with a normalized (Schauder) basis $\left(e_{n}\right)$ and biorthogonal functionals $\left(e_{n}^{*}\right)$. If $A \subset \mathbb{N}$ then $|A|$ denotes the cardinality of $A$ and $1_{A}=\sum_{i \in A} e_{i}$. For $A, B \subset \mathbb{N}$ we write $A<B$ if $\max A<\min B$, if $x \in X$ then we write $\operatorname{supp}(x)=\{n$ : $\left.e_{n}^{*}(x) \neq 0\right\}, P_{A}(x)=\sum_{i \in A} e_{i}^{*}(x) e_{i}$ and $P_{A^{c}}(x)=I-P_{A}(x)$. Set $\mathbb{N}^{m}=\{A \subset \mathbb{N}:|A|=m\}$, $\mathbb{N}^{<\infty}=\cup_{m=0}^{\infty} \mathbb{N}^{m}, \mathcal{A}=\left\{\left(\varepsilon_{i}\right): \varepsilon_{i}=1\right.$ or $\varepsilon_{i}=-1$ for all $\left.i\right\}$ and $1_{\varepsilon A}=\sum_{i \in A} \varepsilon_{i} e_{i}$ for $A \subset \mathbb{N}$, $\varepsilon \in \mathcal{A}$.

The Thresholding Greedy Algorithm (TGA) was introduced by Konyagin and Temlyakov ([7]). This algorithm is defined as follow. For $x \in X$ and $m \in \mathbb{N}$, let $\Lambda_{m}(x)$ be the set of any $m$-indices such that

$$
\min _{n \in \Lambda_{m}(x)}\left|e_{n}^{*}(x)\right| \geq \max _{n \notin \Lambda_{m}(x)}\left|e_{n}^{*}(x)\right| .
$$

Then $G_{m}(x)=\sum_{n \in \Lambda_{m}(x)} e_{n}^{*}(x) e_{n}$ is called an $m$-th greedy approximant to $x$.

Konyagin and Temlyakov in [7] defined a basis $\left(e_{n}\right)$ to be $C$-greedy if for all $x \in X, m \in \mathbb{N}$, we have

$$
\left\|x-G_{m}(x)\right\| \leq C \inf \left\{\left\|x-\sum_{i \in A} a_{i} e_{i}\right\|:|A|=m, a_{i} \in \mathbb{R}, i \in A\right\} .
$$

They showed that greedy bases are characterized by unconditionality and democratic property. Recall that a basis $\left(e_{n}\right)$ of a Banach space $X$ is unconditional if any rearrangement of the series $x=\sum_{n \geq 1} e_{n}^{*}(x) e_{n}$ converges in norm to $x$ for all $x \in X$. A basis $\left(e_{n}\right)$ is said to be

2000 Mathematics Subject Classification. 46B15; 41A65.

Key words and phrases. w-left Property $(A), w$-right Property $(A), w$-partially greedy basis, w-reverse partially greedy basis.

This work was supported by the Israel Science Foundation (Grant Number-137/16). 
democratic if there exists a constant $C \geq 1$ such that $\left\|1_{A}\right\| \leq C\left\|1_{B}\right\|$ where $A, B \in \mathbb{N}<\infty$ and $|A| \leq|B|$.

Konyagin and Temlyakov ([7]) also introduced the notion of quasi-greedy basis. A basis $\left(e_{n}\right)$ of a Banach space $X$ is said to be $C$-quasi-greedy if $\left\|G_{m}(x)\right\| \leq C\|x\|$ for all $x \in X$ and $m \in \mathbb{N}$. Let $C_{q}$ be the least constant among all such $C$. Later, Wojtaszczyk [8] proved that a basis is quasi-greedy if $G_{m}(x) \longrightarrow x$ as $m \longrightarrow \infty$ for $x \in X$.

Dilworth et al. [3] introduced the notions of almost greedy basis and partially greedy basis. A basis $\left(e_{n}\right)$ of a Banach space $X$ is said to be $C$-almost greedy if

$$
\left\|x-G_{m}(x)\right\| \leq C \inf \left\{\left\|x-P_{A}(x)\right\|:|A| \leq m\right\}
$$

for all $x \in X, m \in \mathbb{N}$.

A basis $\left(e_{n}\right)$ is said to be $C$-partially greedy if for all $x \in X$ and $m \in \mathbb{N}$, we have

$$
\left\|x-G_{m}(x)\right\| \leq C\left\|\sum_{n=m+1}^{\infty} e_{i}^{*}(x) e_{i}\right\| .
$$

In [3] the authors proved that almost greedy bases are characterized by quasi-greediness and democracy and partially greedy bases are characterized by quasi-greediness and the conservative property. A basis $\left(e_{n}\right)$ of a Banach space is said to be conservative if there exists a constant $C \geq 1$ such that $\left\|1_{A}\right\| \leq C\left\|1_{B}\right\|$ whenever $A<B$ and $|A| \leq|B|$.

If $x \in X$ then a greedy ordering for $x$ is a $1-1$ map $\rho: \mathbb{N} \longrightarrow \mathbb{N}$ such that $\operatorname{supp}(x) \subset \rho(\mathbb{N})$ and if $j<k$, then $\left|e_{\rho(j)}^{*}(x)\right| \geq\left|e_{\rho(k)}^{*}(x)\right|$. Note that if $x$ has infinite support and the nonzero basis coefficients of $x$ are distinct then $x$ has a unique greedy ordering.

If $\rho$ is a greedy ordering for $x \in X$, then $\Lambda_{m}(x)=\{\rho(1), \cdots, \rho(m)\}$ and we denote $\alpha_{m}(x)=$ $\min \Lambda_{m}(x), \beta_{m}(x)=\max \Lambda_{m}(x)$.

Recently, in [4], the authors gave another characterization of partially greedy basis and introduced the notion of reverse partially greedy basis. They proved that a basis $\left(e_{n}\right)$ is partially greedy if and only if there exists a constant $C$ such that

$$
\left\|x-G_{m}(x)\right\| \leq C \inf \left\{\left\|x-P_{A}(x)\right\|:|A| \leq m, A<\alpha_{m}(x)\right\}
$$

for all $x \in X$ and $m \in \mathbb{N}$.

Throughout this paper we will use this characterization of the partially greedy bases from [4.

A basis $\left(e_{n}\right)$ is said to be $C$-reverse partially greedy if for all $x \in X$ and $m \in \mathbb{N}$, we have

$$
\left\|x-G_{m}(x)\right\| \leq C \inf \left\{\left\|x-P_{A}(x)\right\|:|A| \leq m, A>\beta_{m}(x)\right\} .
$$

A weight sequence $w=\left(w_{n}\right)$ is a sequence of positive real numbers. For any $A \subset \mathbb{N}$ we write $w(A)=\sum_{i \in A} w_{i}$.

In [6] the authors studied weight-greedy bases. Recently, in [5] and [2], the authors studied weight-almost greedy bases and weight-partially greedy bases (using the definition from [3]). In [6], 5] and [2] the authors proved a criterion for weight-greedy, weight-almost greedy and 
weight-partially greedy bases similar to the one for greedy, almost greedy and partially greedy bases respectively. In [2] the notion of $w$-Property $(A)$ was introduced and a characterization of $(i) w$-greedy bases in terms of unconditionality and w-Property $(A)(i i) w$-almost greedy bases in terms of quasi-greediness and $w$-Property $(A)$ was proved.

In this paper we will introduce the notion of $w$-partially greedy bases (using the characterization (2) ) and $w$-reverse partially greedy bases. In section 2 we will prove that a basis is $w$-partially greedy ( $w$-reverse partially greedy) if and only if it is both quasi-greedy and $w$-conservative ( $w$-reverse conservative). In section 3 we will introduce the notion of $w$-left Property $(A)$ and $w$-right Property $(A)$. We will prove another characterization of $w$-partially greedy ( $w$-reverse partially greedy) bases, precisely, we will prove that a basis is $w$-partially greedy ( $w$-reverse partially greedy) if and only if it is quasi-greedy and satisfies $w$-left Property $(A)$ (w-right Property $(A))$. In this section, we will also consider some conditions on the weight sequences such that any basis satisfying both $w$-left Property $(A)$ and $w$-right Property $(A)$ satisfies $w$-Property $(A)$. In the last section we will study $w$-conservative and $w$-reverse conservative basis. $w$-conservative and $w$-reverse conservative properties are weaker conditions than $w$-left Property $(A)$ and $w$-right Property $(A)$ respectively.

Let

$$
\widetilde{\sigma}_{w\left(\Lambda_{m}(x)\right)}^{L}(x)=\inf \left\{\left\|x-P_{A}(x)\right\|: A \in \mathbb{N}^{<\infty}, w(A) \leq w\left(\Lambda_{m}(x)\right), A<\alpha_{m}(x)\right\}
$$

and

$$
\widetilde{\sigma}_{w\left(\Lambda_{m}(x)\right)}^{R}(x)=\inf \left\{\left\|x-P_{A}(x)\right\|: A \in \mathbb{N}^{<\infty}, w(A) \leq w\left(\Lambda_{m}(x)\right), \beta_{m}(x)<A\right\}
$$

for any $x \in X, m \in \mathbb{N}$.

Definition 1.1. We say that a basis $\left(e_{n}\right)$ is $C$-w-partially greedy if

$$
\left\|x-G_{m}(x)\right\| \leq C \tilde{\sigma}_{w\left(\Lambda_{m}(x)\right)}^{L}(x)
$$

for all $x \in X, m \in \mathbb{N}$.

Definition 1.2. We say that a basis $\left(e_{n}\right)$ is $C$-w-reverse partially greedy if

$$
\left\|x-G_{m}(x)\right\| \leq C \tilde{\sigma}_{w\left(\Lambda_{m}(x)\right)}^{R}(x)
$$

for all $x \in X, m \in \mathbb{N}$.

Let $C_{p}, C_{r p}$ be the least constants satisfying (6) and (7) respectively.

Definition 1.3. A basis $\left(e_{n}\right)$ of a Banach space $X$ is said to be $w$-democratic if there exists a constant $C \geq 1$ such that $\left\|1_{A}\right\| \leq C\left\|1_{B}\right\|$ whenever $A, B \in \mathbb{N}^{<\infty}$ and $w(A) \leq w(B)$.

Definition 1.4. A basis $\left(e_{n}\right)$ of a Banach space $X$ is said to be $w$-conservative $(w$-reverse conservative) if there exists a constant $C \geq 1$ such that $\left\|1_{A}\right\| \leq C\left\|1_{B}\right\|$ whenever $A, B \in$ $\mathbb{N}<\infty, A<B(B<A)$ and $w(A) \leq w(B)$. 
If $w=(1,1,1, \cdots)$ then $w(A)=|A|$ for any $A \in \mathbb{N}^{<\infty}$ and in this case $w$-partially greedy ( $w$-reverse partially greedy, $w$-democratic, $w$-conservative, $w$-reverse conservative) basis is partially greedy (reverse partially greedy, democratic, conservative, reverse conservative) basis.

We now recall a few results related to quasi-greedy bases from [3].

Lemma 1.5. Suppose that $\left(e_{n}\right)$ is a $K$-quasi-greedy basis and $A \in \mathbb{N}<\infty$. Then, for every choice of scalars $\left(a_{i}\right)_{i \in A}$ we have

$$
\left\|\sum_{j \in A} a_{j} e_{j}\right\| \leq 2 K \max _{j \in A}\left|a_{j}\right|\left\|\sum_{j \in A} e_{j}\right\| .
$$

Lemma 1.6. Suppose that $\left(e_{n}\right)$ is a $K$-quasi-greedy basis and $x \in X$ has greedy ordering $\rho$. Then

$$
\left|e_{\rho(m)}^{*}(x)\right|\left\|\sum_{1}^{m} e_{\rho(i)}\right\| \leq 4 K^{2}\|x\| .
$$

\section{WEIGHT-PARTIALLY AND WEIGHT-REVERSE PARTIALLY GREEDY BASES}

Theorem 2.1. Suppose $\left(e_{n}\right)$ is a basis of a Banach space $X$. Then the following assertions are equivalent:

(a) $\left(e_{n}\right)$ is w-partially greedy.

(b) $\left(e_{n}\right)$ is quasi-greedy and $w$-conservative.

Proof. We first prove that $(a)$ implies $\left(e_{n}\right)$ is $w$-conservative. Let $\left(e_{n}\right)$ be $C_{p}$-w-partially greedy, $A, B \in \mathbb{N}^{<\infty}$ with $A<B$ and $w(A) \leq w(B)$. Consider $x=1_{A}+(1+\varepsilon) 1_{B}$ for any $\varepsilon>0$. Then $G_{|B|}(x)=(1+\varepsilon) 1_{B}$. Now from (6) we have $\left\|1_{A}\right\| \leq C_{p} \widetilde{\sigma}_{w\left(\Lambda_{|B|}(x)\right)}^{L}(x) \leq$ $C_{p}(1+\varepsilon)\left\|1_{B}\right\|$. Thus, by letting $\varepsilon \rightarrow 0$, we get $\left(e_{n}\right)$ is $w$-conservative. Clearly $(a)$ implies that $\left\|x-G_{m}(x)\right\| \leq C_{p}\|x\|$ and hence the basis is quasi-greedy.

Now we will prove that $(b)$ implies $(a)$. Let the basis $\left(e_{n}\right)$ be $C_{q}$-quasi-greedy and $w$ conservative with constant $C$. Let $x \in X, m \in \mathbb{N}$ and $G_{m}(x)=\sum_{i \in \Lambda_{m}(x)} e_{i}^{*}(x) e_{i}$.

If $A \in \mathbb{N}^{<\infty}$ with $A<\alpha_{m}(x)$ and $w(A) \leq w\left(\Lambda_{m}(x)\right)$, then

$$
x-G_{m}(x)=x-\sum_{i \in A} e_{i}^{*}(x) e_{i}-\sum_{i \in \Lambda_{m}(x)} e_{i}^{*}(x) e_{i}+\sum_{i \in A} e_{i}^{*}(x) e_{i} .
$$

We can write

$$
\sum_{i \in \Lambda_{m}(x)} e_{i}^{*}(x) e_{i}=G_{m}\left(x-\sum_{i \in A} e_{i}^{*}(x) e_{i}\right)
$$

and hence

$$
\left\|\sum_{i \in \Lambda_{m}(x)} e_{i}^{*}(x) e_{i}\right\| \leq C_{q}\left\|x-\sum_{i \in A} e_{i}^{*}(x) e_{i}\right\|
$$


From (8), (9) and the $w$-conservative property we have

$$
\begin{aligned}
\left\|\sum_{i \in A} e_{i}^{*}(x) e_{i}\right\| & \leq 2 C_{q} \max _{i \in A}\left|e_{i}^{*}(x)\right|\left\|1_{A}\right\| \\
& \leq 2 C_{q} C \min _{i \in \Lambda_{m}(x)}\left|e_{i}^{*}(x)\right|\left\|1_{\Lambda_{m}(x)}\right\| \\
& \leq 8 C_{q}^{3} C\left\|\sum_{i \in \Lambda_{m}(x)} e_{i}^{*}(x) e_{i}\right\| \\
& \leq 8 C_{q}^{4} C\left\|x-\sum_{i \in A} e_{i}^{*}(x) e_{i}\right\|
\end{aligned}
$$

Thus $\left\|x-G_{m}(x)\right\| \leq\left(1+C_{q}+8 C_{q}^{4} C\right)\left\|x-\sum_{i \in A} e_{i}^{*}(x) e_{i}\right\|$.

Remark 2.2. In [2] a basis $\left(e_{n}\right)$ of a Banach space $X$ was defined to be $w$-partially greedy if for all $m, r$ such that $w(\{1, \cdots, m\}) \leq w\left(\Lambda_{r}(x)\right)$, there exists a constant $C$ such that

$$
\left\|x-G_{r}(x)\right\| \leq C\left\|\sum_{m+1}^{\infty} e_{i}^{*}(x) e_{i}\right\|
$$

It was proved in 2] that a basis is $w$-partially greedy if and only if it is quasi-greedy and $w$ conservative. Thus from Theorem 2.1 it follows that the $w$-partially greedy basis considered in this paper is equivalent to the one considered in [2].

Similar arguments as in Theorem 2.1 yields the following result.

Theorem 2.3. Suppose $\left(e_{n}\right)$ is a basis of a Banach space $X$. Then the following assertions are equivalent:

(a) $\left(e_{n}\right)$ is w-reverse partially greedy.

(b) $\left(e_{n}\right)$ is quasi-greedy and w-reverse conservative.

3. WEIGHT-LEFT PROPERTY (A) AND WEIGHT-RIGHT PROPERTY (A)

Following notion of $w$-Property $(A)$ was introduced in [2].

Definition 3.1. A basis $\left(e_{n}\right)$ of a Banach space $X$ is said to have $w$-Property $(A)$ if there exists a constant $C \geq 1$ such that

$$
\left\|x+t 1_{\varepsilon A}\right\| \leq C\left\|x+t 1_{\eta B}\right\|
$$

for any $x \in X, t \geq \sup _{j}\left|e_{j}^{*}(x)\right|, A, B \in \mathbb{N}<\infty, A \cap B=\emptyset, w(A) \leq w(B), \operatorname{supp}(x) \cap(A \cup B)=\emptyset$ and $\varepsilon, \eta \in \mathcal{A}$.

Motivated by this notion of $w$-Property $(A)$, we now give the definitions of $w$-left Property $(A)$ and $w$-right Property $(A)$.

Definition 3.2. We say a basis $\left(e_{n}\right)$ of a Banach space $X$ satisfies $w$-left Property $(A)$ if there exists a constant $C \geq 1$ such that

$$
\left\|x+t 1_{\varepsilon A}\right\| \leq C\left\|x+t 1_{\eta B}\right\|
$$


for any $x \in X, t \geq \sup _{j}\left|e_{j}^{*}(x)\right|, A, B \in \mathbb{N}<\infty$ with $A<B, w(A) \leq w(B), \operatorname{supp}(x) \cap(A \cup B)=$ $\emptyset$ and $\varepsilon, \eta \in \mathcal{A}$.

Definition 3.3. A basis $\left(e_{n}\right)$ of a Banach space $X$ is said to have $w$-right Property $(A)$ if there exists a constant $C \geq 1$ such that

$$
\left\|x+t 1_{\varepsilon A}\right\| \leq C\left\|x+t 1_{\eta B}\right\|
$$

for any $x \in X, t \geq \sup _{j}\left|e_{j}^{*}(x)\right|, A, B \in \mathbb{N}^{<\infty}$ with $B<A, w(A) \leq w(B), \operatorname{supp}(x) \cap(A \cup B)=$ $\emptyset$ and $\varepsilon, \eta \in \mathcal{A}$.

Let $C_{a}, C_{l a}, C_{r a}$ be the least constants satisfying (10), (11) and (12) respectively. We will write $\left(e_{n}\right)$ satisfies $C_{a}$-w-Property $(A), C_{l a}$-w-left Property $(A)$ and $C_{r a}$-w-right Property $(A)$.

Remark 3.4. Observe that any basis satisfying $w$-Property $(A), w$-left Property $(A), w$-right Property $(A)$ is respectively $w$-democratic, $w$-conservative and $w$-reverse conservative.

For $w=(1,1,1, \cdots)$ we will write $w$-Property $(A)$ (w-left Property $(A), w$-right Property $(A))$ as Property $(A)$ (left Property $(A)$, right Property $(A)$ ). While considering $w$-right Property $(A)$ and $w$-reverse conservative bases for any $B \in \mathbb{N}<\infty$, we will always include the empty set in the collection $\{A \in \mathbb{N}<\infty: B<A, w(A) \leq w(B)\}$.

Remark 3.5. Albiac and Wojtaszczyk introduced the following notion of Property $(A)$ (we will refer to it as classical Property $(A))$ in [1] to study a characterization of 1-greedy basis. A basis $\left(e_{n}\right)$ has classical Property $(A)$ if there exists a constant $C \geq 1$ such that

$$
\left\|x+t 1_{\varepsilon A}\right\| \leq C\left\|x+t 1_{\eta B}\right\|
$$

for any $x \in X, t \geq \sup _{j}\left|e_{j}^{*}(x)\right|, A, B \in \mathbb{N}<\infty, A \cap B=\emptyset,|A|=|B|, \operatorname{supp}(x) \cap(A \cup B)=\emptyset$ and $\varepsilon, \eta \in \mathcal{A}$. In this paper we are dealing only with the Schauder basis so the Property $(A)$ is equivalent to the classical Property $(A)$.

Example 3.6. We present examples of basis which satisfy left Property $(A)$ or right Property (A) but does not satisfy Property $(A)$.

(a) Let $p=\left(p_{n}\right)$ be a strictly increasing sequence of natural numbers and $M_{n}(t)=t^{p_{n}}$ for $t \geq 0$. Let $X_{p}$ be the corresponding modular sequence space, that is, $X_{p}$ is the Banach space consisting of all sequences $x=\left(x_{n}\right)$ with $\sum\left(\frac{\left|x_{n}\right|}{\lambda}\right)^{p_{n}}<\infty$ for some $\lambda>0$ and the norm on $X_{p}$ is defined as

$$
\|x\|_{p}=\inf \left\{\lambda>0: \sum\left(\frac{\left|x_{n}\right|}{\lambda}\right)^{p_{n}} \leq 1\right\} .
$$

Let $x \in X, t \geq \sup _{j}\left|e_{j}^{*}(x)\right|, A, B \in \mathbb{N}<\infty$ with $B<A,|A| \leq|B|, \operatorname{supp}(x) \cap(A \cup B)=$ $\emptyset$ and $\varepsilon, \eta \in \mathcal{A}$. Then clearly $\left\|x+t 1_{\varepsilon A}\right\|_{p} \leq\left\|x+t 1_{\eta B}\right\|_{p}$, thus the canonical basis $\left(e_{n}\right)$ of $X_{p}$ satisfies right Property $(A)$ and its easy to observe that $\left(e_{n}\right)$ does not satisfy left Property $(A)$. 
(b) For each $n \in \mathbb{N}$, we define $F_{n}:=\{A \subset \mathbb{N}:|A| \leq n$ !, $n ! \leq A\}$ and $F:=\bigcup_{n>1} F_{n}$. Observe that the set $F$ is closed under spreading to the right: in fact, if $A, B \in \mathbb{N}^{m}$, $A \in F$ and $\min A \leq \min B$, then $B \in F$.

We define a norm on $c_{00}$ (the space of all sequences of real numbers with finitely many non-zero terms) as follows

$$
\|x\|=\max \left\{\left(|x|, 1_{A}\right): A \in F\right\}
$$

for $x \in c_{00}$. Let $X$ be the completion of $c_{00}$ in this norm. The canonical basis $\left(e_{n}\right)$ of $X$ is normalized and 1-unconditional.

From the right spreading property of $F$, it follows that $\|x\| \leq\|y\|$, where $y=\sum a_{i} e_{n_{i}}$ is a spread of $x=\sum a_{i} e_{i}$ with $n_{1}<n_{2}<\ldots$ In particular, if $A<B$ and $|A| \leq|B|$ then for any $x \in X, t \geq \sup _{j}\left|e_{j}^{*}(x)\right|$, supp $(x) \cap(A \cup B)=\emptyset$ and $\varepsilon, \eta \in \mathcal{A}$ we get $\left\|x+t 1_{\varepsilon A}\right\| \leq\left\|x+t 1_{\eta B}\right\|$. Thus $\left(e_{n}\right)$ satisfies left Property $(A)$.

Observe that, $\left\|1_{[1, n !]}\right\|=(n-1)$ !, while $\left\|1_{[n !+1,2 n !]}\right\|=n$ !. So $\left(e_{n}\right)$ is not a democratic basis and hence $\left(e_{n}\right)$ cannot satisfy Property $(A)$.

Now consider the dual norm $\|\cdot\|^{*}$. Since $\|x\| \leq\|y\|$ it follows easily that $\|x\|^{*} \geq$ $\|y\|^{*}$. In particular, $\|\cdot\|^{*}$ satisfies right Property $(A)$. But in this case $\left\|1_{[1, n !]}\right\|=$ $(n-1)$ ! implies $\left\|1_{[1, n !]}\right\|^{*} \geq n$ while $\left\|1_{[n !+1,2 n !]}\right\|^{*}=1$ since $[n !+1,2 n !] \in F_{n}$. So the dual norm is not democratic and hence it cannot satisfy Property $(A)$.

Example 3.7. Now we shall present an example of a basis which is conservative but does not satisfies left Property $(A)$. Let $\left(a_{i}\right)$ be a sequence of natural numbers such that

$$
a_{2 i}=i \text { and } a_{2 i-1}=i \text { for } i \in \mathbb{N} \text {. }
$$

We define a norm on $c_{00}$ as follows

$$
\|x\|=\sup _{N}\left|\sum_{1}^{N} a_{i} x_{i}\right|
$$

for $x \in c_{00}$. Let $X$ be the completion of $c_{00}$ in this norm. The canonical basis $\left(e_{n}\right)$ of $X$ is normalized basis. Clearly $\left(e_{n}\right)$ is conservative but is not a democratic basis.

Let $n \in \mathbb{N}$ be any even number, $A=\{n+1, \cdots, 2 n\}$ and $B=\{2 n+2,2 n+4, \cdots, 4 n-2,4 n\}$. Choose $x \in X$ with $\operatorname{supp}(x)=\{2 n+1,2 n+3, \cdots, 4 n-3,4 n-1\}$ and $x_{i}=-1$ for all $i \in \operatorname{supp}(x)$. Now for any $\varepsilon, \eta \in \mathcal{A}$ with $\varepsilon_{i}, \eta_{i}=1$ for all $i$ we get

$$
\left\|x+1_{\varepsilon A}\right\|=\frac{n}{2}\left(\frac{3 n}{2}+1\right)
$$

and

$$
\left\|x+1_{\eta B}\right\|=2 n
$$

Thus $\left(e_{n}\right)$ does not satisfies left Property $(A)$. 
We now give characterization of $w$-partially greedy bases in terms of quasi-greedy property and $w$-left Property $(A)$.

Proposition 3.8. Let $\left(e_{n}\right)$ be a basis of a Banach space $X$. If $\left(e_{n}\right)$ is $C_{p}$-w-partially greedy, then $\left(e_{n}\right)$ is $\left(C_{p}+1\right)$-quasi-greedy and has $C_{p}$-w-left Property $(A)$.

Proof. Let $\left(e_{n}\right)$ be a $w$-partially greedy basis with constant $C_{p}$. Thus

$$
\left\|x-G_{m}(x)\right\| \leq C_{p} \inf \left\{\left\|x-P_{A}(x)\right\|: A \in \mathbb{N}^{<\infty}, w(A) \leq w\left(\Lambda_{m}(x)\right), A<\alpha_{m}(x)\right\}
$$

for all $x \in X$ and $m \in \mathbb{N}$.

If we take $A=\emptyset$, then we get $\left\|x-G_{m}(x)\right\| \leq C_{p}\|x\|$ and hence $\left\|G_{m}(x)\right\| \leq\left(C_{p}+1\right)\|x\|$ for all $x \in X, m \in \mathbb{N}$.

Let $x \in X, t \geq \sup _{j}\left|e_{j}^{*}(x)\right|, A, B \in \mathbb{N}<\infty$ with $A<B, w(A) \leq w(B), \operatorname{supp}(x) \cap(A \cup B)=\emptyset$ and $\varepsilon, \eta \in \mathcal{A}$. If we take $y=x+t 1_{\varepsilon A}+(t+\delta) 1_{\eta B}$ for any $\delta>0$, then $\left\|x+t 1_{\varepsilon A}\right\|=$ $\left\|y-G_{|B|}(y)\right\| \leq C_{p}\left\|y-t 1_{\varepsilon A}\right\|=\left\|x+(t+\delta) 1_{\eta B}\right\|$. This gives that $\left(e_{n}\right)$ satisfies $C_{p^{-}}-w$-left Property $(A)$.

From Remark 3.4 it is clear that $w$-left Property $(A)$ implies $w$-conservative property. Thus from Theorem 2.1 it follows that if a basis $\left(e_{n}\right)$ is quasi-greedy and has $w$-left Property $(A)$ then $\left(e_{n}\right)$ is $w$-partially greedy. We now prove this result using the arguments similar to [2] to get better estimates in terms of the constant.

Proposition 3.9. A basis $\left(e_{n}\right)$ of a Banach space $X$ satisfies w-left left Property $(A)$ with constant $C_{l a}$ if and only if

$$
\|x\| \leq C_{l a}\left\|x-P_{A}(x)+1_{\eta B}\right\|
$$

where $x \in X, \sup _{j}\left|e_{j}^{*}(x)\right| \leq 1, A, B \in \mathbb{N}<\infty, A<B, w(A) \leq w(B)$, supp $(x) \cap B=\emptyset$ and $\eta \in \mathcal{A}$.

Proof. First observe that [2, Lemma 2.4] implies for the proof of the result it is sufficient to consider only finitely supported $x \in X$.

Let $\left(e_{n}\right)$ has $w$-left Property $(A)$ with constant $C_{l a}, x \in X, \sup _{j}\left|e_{j}^{*}(x)\right| \leq 1, A, B \in \mathbb{N}<\infty$, $A<B, w(A) \leq w(B), \operatorname{supp}(x) \cap B=\emptyset$ and $\varepsilon, \eta \in \mathcal{A}$. From the $w$-left Property $(A)$ of the basis we can write

$$
\begin{aligned}
\left\|P_{A^{c}}(x)+1_{\varepsilon A}\right\| & \leq C_{l a}\left\|P_{A^{c}}(x)+1_{\eta B}\right\| \\
& =C_{l a}\left\|x-P_{A}(x)+1_{\eta B}\right\| .
\end{aligned}
$$

Since $x$ belongs to the convex hull of $\left\{P_{A^{c}}(x)+1_{\varepsilon A}: \varepsilon \in \mathcal{A}\right\}$, so we get

$$
\|x\| \leq C_{l a}\left\|x-P_{A}(x)+1_{\eta B}\right\| .
$$


Conversely, for any $x \in X$ with $\sup _{j}\left|e_{j}^{*}(x)\right| \leq 1, A, B \in \mathbb{N}<\infty, A<B, w(A) \leq w(B)$, $\operatorname{supp}(x) \cap(A \cup B)=\emptyset$ and $\varepsilon, \eta \in \mathcal{A}$, consider $y=x+1_{\varepsilon A}$. Now from (13) we get

$$
\begin{aligned}
\|y\|=\left\|x+1_{\varepsilon A}\right\| & \leq C_{l a}\left\|y-P_{A}(y)+1_{\eta B}\right\| \\
& =C_{l a}\left\|x+1_{\eta B}\right\| .
\end{aligned}
$$

Similarly we can prove the following results for a basis with $w$-right Property $(A)$.

Proposition 3.10. A basis $\left(e_{n}\right)$ of a Banach space $X$ satisfies $C_{r a}$-w-right Property $(A)$ if and only if

$$
\|x\| \leq C_{r a}\left\|x-P_{A}(x)+1_{\eta B}\right\|
$$

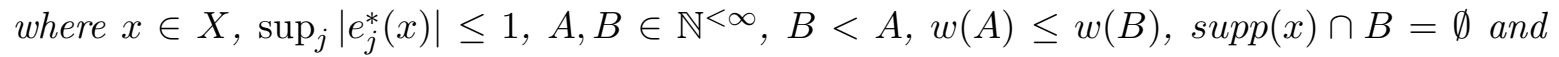
$\eta \in \mathcal{A}$.

Before proving the next result we first recall the following result from [2].

Lemma 3.11. Let $\left(e_{n}\right)$ be a $C_{q}$-quasi-greedy basis of a Banach space $X, x \in X$ and $\lambda>0$. If $T_{\lambda}$ is an operator defined on $X$ by

$$
T_{\lambda}(x)=\sum_{i \in A} \lambda \operatorname{sgn}\left(e_{i}^{*}(x)\right) e_{i}+\sum_{i \in A^{c}} e_{i}^{*}(x) e_{i}
$$

where $A=\left\{i: \lambda<\left|e_{i}^{*}(x)\right|\right\}$, then $\left\|T_{\lambda}(x)\right\| \leq\left(C_{q}+1\right)\|x\|$.

Theorem 3.12. Let $\left(e_{n}\right)$ be a basis of a Banach space $X$.

(a) If $\left(e_{n}\right)$ is $C_{p}$-w-partially greedy, then $\left(e_{n}\right)$ is $\left(C_{p}+1\right)$-quasi-greedy and has $C_{p}$-w-left Property $(A)$.

(b) If $\left(e_{n}\right)$ has $C_{l a}-w$-left Property $(A)$ and is $C_{q^{-}}$quasi-greedy then $\left(e_{n}\right)$ is $\left(C_{q}+1\right) C_{l a^{-}}$ $w$-partially greedy.

Proof. (a) follows from Proposition 3.8 .

Let $x \in X$ and $\varepsilon>0$. Now, choose $A \in \mathbb{N}^{<\infty}$ with $A<\Lambda_{m}(x), w(A) \leq w\left(\Lambda_{m}(x)\right)$ such that $\left\|x-P_{A}(x)\right\|<\tilde{\sigma}_{w\left(\Lambda_{m}(x)\right)}^{L}(x)+\varepsilon$. Fix $t=\left|e_{\rho(m)}^{*}(x)\right|$ and $\eta \in \mathcal{A}$ with $\eta_{j}=\operatorname{sgn}\left(e_{j}^{*}(x)\right)$ for all $j$, then from Proposition 3.9 and Lemma 3.11 it follows that

$$
\begin{aligned}
\left\|x-G_{m}(x)\right\| & \leq C_{l a}\left\|x-P_{\Lambda_{m}(x)}-P_{A}(x)+t 1_{\eta \Lambda_{m}(x)}\right\| \\
& =C_{l a}\left\|P_{\left(A \cup \Lambda_{m}(x)\right)^{c}}\left(x-P_{A}(x)\right)+t 1_{\eta \Lambda_{m}(x)}\right\| \\
& =C_{l a}\left\|T_{t}\left(I-P_{A}\right)(x)\right\| \\
& \leq\left(C_{q}+1\right) C_{l a}\left\|x-P_{A}(x)\right\| \\
& <\left(C_{q}+1\right) C_{l a}\left(\tilde{\sigma}_{w\left(\Lambda_{m}(x)\right)}^{L}(x)+\varepsilon\right) .
\end{aligned}
$$

From this we can conclude that $\left\|x-G_{m}(x)\right\| \leq\left(C_{q}+1\right) C_{l a} \widetilde{\sigma}_{w\left(\Lambda_{m}(x)\right)}^{L}(x)$ and thus the basis is $\left(C_{q}+1\right) C_{l a}-w$-partially greedy. 
In a similar fashion we can prove the following characterization of $w$-reverse partially greedy basis.

Theorem 3.13. Let $\left(e_{n}\right)$ be a basis of a Banach space $X$.

(a) If $\left(e_{n}\right)$ is $C_{r p}$-w-reverse partially greedy, then $\left(e_{n}\right)$ is $\left(C_{r p}+1\right)$-quasi-greedy and has $C_{r p}$-w-left Property $(A)$.

(b) If $\left(e_{n}\right)$ has $C_{r a}$-w-right Property $(A)$ and is $C_{q}$-quasi-greedy then $\left(e_{n}\right)$ is $\left(C_{q}+1\right) C_{r a^{-}}$ $w$-reverse partially greedy.

We now prove that if a basis $\left(e_{n}\right)$ of a Banach space $X$ satisfies both left Property $(A)$ and right Property $(A)$ then the basis satisfies Property $(A)$. If $X$ is an infinite-dimensional Banach space then for given $A, B \in \mathbb{N}^{m}$ we can find $C \in \mathbb{N}^{m}$ with $A<C$ and $B<C$. Now by the left Property $(A)$ and right Property $(A)$ of the basis it can be easily proved that the basis satisfies Property $(A)$. We now give another proof of this fact which has the advantage of working for a finite basis as well.

Lemma 3.14. Let $\left(e_{n}\right)$ be a basis of Banach space $X$. If $\left(e_{n}\right)$ satisfies both left Property $(A)$ and right Property $(A)$ then the basis satisfies Property $(A)$.

Proof. Let $C$ be a constant such that for any $x \in X, t \geq \sup _{n}\left|e_{n}^{*}(x)\right|, \varepsilon, \eta \in \mathcal{A}, A, B \in \mathbb{N}<\infty$, $\operatorname{supp}(x) \cap(A \cup B)=\emptyset$ and $|A| \leq|B|$ we have

$$
\left\|x+t 1_{\varepsilon A}\right\| \leq C\left\|x+t 1_{\eta B}\right\|
$$

where either $A<B$ or $B>A$.

Observe that to prove Property $(A)$ it is sufficient to consider any two disjoint sets $A, B$ of same cardinality. Choose any $x \in X, t \geq \sup _{n}\left|e_{n}^{*}(x)\right|, \varepsilon, \eta \in \mathcal{A}$ and any two disjoint sets $A, B \in \mathbb{N}^{m}$ with $\operatorname{supp}(x) \cap(A \cup B)=\emptyset$. Let $A=\left\{a_{1}<a_{2}<\ldots<a_{m}\right\}$ and $B=\left\{b_{1}<b_{2}<\right.$ $\left.\ldots<b_{m}\right\}$. If $A<B$ or $B<A$, then $\left\|x+t 1_{\varepsilon A}\right\| \leq C\left\|x+t 1_{\eta B}\right\|$ and $\left\|x+t 1_{\eta B}\right\| \leq C\left\|x+t 1_{\varepsilon A}\right\|$. So, for the rest of the proof we assume that this is not the case.

Without loss of generality we can assume that $a_{1}<b_{m}$. If we compare $a_{2}$ with $b_{m-1}$ then there can be two possibilities: $a_{2}<b_{m-1}$ or $a_{2}>b_{m-1}$. If $a_{2}>b_{m-1}$ then we will stop the process; otherwise we will continue in the same manner. By the assumptions on the sets $A, B$ we can find the first $j, 1 \leq j<m$, such that $a_{j+1}>b_{m-j}$.

Thus we can write

$A=A_{1} \cup A_{2}$ and $B=B_{1} \cup B_{2}$ where $\left|A_{i}\right|=\left|B_{i}\right|, A_{1}<B_{1}, A_{2}>B_{2}, A_{1}=\left\{a_{1}<\ldots<\right.$ $\left.a_{j}\right\}<B_{1}=\left\{b_{m-j+1}<\ldots<b_{m}\right\}$ and $A_{2}=\left\{a_{j+1}<\ldots<a_{m}\right\}>B_{2}=\left\{b_{1}<\ldots<b_{m-j}\right\}$

Let $x_{1}=\sum_{i>\min B_{1}} e_{i}^{*}(x) e_{i}, x_{2}=x-x_{1}, y_{1}=\sum_{i<\max A_{1}} e_{i}^{*}(x) e_{i}$ and $y_{2}=x-y_{1}$. Then

$$
\left\|x_{i}+t 1_{\varepsilon A_{i}}\right\| \leq C\left\|x_{i}+t 1_{\eta B_{i}}\right\|, i=1,2
$$

and

$$
\left\|y_{i}+t 1_{\eta B_{i}}\right\| \leq C\left\|y_{i}+1_{\varepsilon A_{i}}\right\|, \quad i=1,2
$$


Now we can write

$$
\begin{aligned}
\left\|x+t 1_{\varepsilon A}\right\| & \leq\left\|x_{1}+t 1_{\varepsilon A_{1}}\right\|+\left\|x_{2}+t 1_{\varepsilon A_{2}}\right\| \\
& \leq C\left(\left\|x_{1}+t 1_{\eta B_{1}}\right\|+\left\|x_{2}+t 1_{\eta B_{2}}\right\|\right) \\
& \leq C\left(2 K_{b}+1\right)\left\|x+t 1_{\eta B}\right\|
\end{aligned}
$$

and

$$
\begin{aligned}
\left\|x+t 1_{\eta B}\right\| & \leq\left\|y_{1}+t 1_{\eta B_{1}}\right\|+\left\|y_{2}+t 1_{\eta B_{2}}\right\| \\
& \leq C\left(\left\|y_{1}+t 1_{\varepsilon A_{1}}\right\|+\left\|y_{2}+t 1_{\varepsilon A_{2}}\right\|\right) \\
& \leq C\left(2 K_{b}+1\right)\left\|x+t 1_{\varepsilon A}\right\|
\end{aligned}
$$

where $K_{b}$ is the basis constant for $\left(e_{n}\right)$.

Consider any weight sequence $w=\left(w_{n}\right)$ bounded away from 0 and $\infty$. For such a weight sequence we now prove that any basis satisfying both $w$-left Property $(A)$ and $w$-right Property $(A)$ satisfies $w$-Property $(A)$. If $X$ is an infinite-dimensional Banach space then this result can be proved by a simple argument. But we will present a proof which works for both finite and infinite-dimensional Banach spaces.

To prove this result we first extend the idea used in [5, Proposition 4.9] to prove that in this case any basis $\left(e_{n}\right)$ has $w$-left Property $(A)$ (w-right Property $\left.(A)\right)$ if and only if $\left(e_{n}\right)$ has left Property $(A)$ (right Property $(A)$ ).

Proposition 3.15. Let $w$ be a weight sequence with $0<\inf w_{n} \leq \sup w_{n}<\infty$. Then $\left(e_{n}\right)$ has w-left Property $(A)$ if and only if $\left(e_{n}\right)$ has left Property $(A)$.

Proof. For simplicity we assume that $0<\alpha=\inf w_{n} \leq \sup w_{n}=1$.

Let $\left(e_{n}\right)$ satisfies $w$-left Property $(A)$ with constant $C_{l a}$. Let $x \in X, t \geq \sup _{n}\left|e_{n}^{*}(x)\right|, \varepsilon, \eta \in$ $\mathcal{A}, A, B \in \mathbb{N}^{<\infty}$ with $A<B,|A|=|B|$ and $\operatorname{supp}(x) \cap(A \cup B)=\emptyset$.

If $w(A) \leq w(B)$ then from the $w$-left Property $(A)$ of the basis we get

$$
\left\|x+t 1_{\varepsilon A}\right\| \leq C_{l a}\left\|x+t 1_{\eta B}\right\| .
$$

Now consider the case when $w(B) \leq w(A)$. If in this case $w(A) \leq \frac{2}{\alpha}$ then $|A|,|B| \leq \frac{2}{\alpha^{2}}$ and thus

$$
\begin{aligned}
\left\|x+t 1_{\varepsilon A}\right\| & \leq\left\|x+t 1_{\eta B}\right\|+\left\|t 1_{\varepsilon A}\right\|+\left\|t 1_{\eta B}\right\| \\
& \leq\left\|x+t 1_{\eta B}\right\|+\frac{4}{\alpha^{2}} t \\
& =\left\|x+t 1_{\eta B}\right\|+\frac{4}{\alpha^{2}}\left\|t 1_{\eta b}\right\| \\
& \leq\left\|x+t 1_{\eta B}\right\|+\frac{8 K_{b}}{\alpha^{2}}\left\|x+t 1_{\eta B}\right\|
\end{aligned}
$$

where $K_{b}$ is the basis constant of $\left(e_{n}\right)$ and $b \in B$. 
If $w(A)>\frac{2}{\alpha}$ then $2<\alpha w(A) \leq \alpha|A|=\alpha|B| \leq w(B)$. Thus we can partition $A$ into $N$ sets $A_{1}, \ldots, A_{N}$ satisfying $w\left(A_{i}\right) \leq w(B) \leq w\left(A_{i}\right)+1$, hence $w\left(A_{i}\right) \geq \frac{w(B)}{2}$ and

$$
N \leq \frac{w(A)}{\frac{w(B)}{2}} \leq \frac{2}{\alpha} .
$$

Now by combining the facts that $A_{i}<B, w\left(A_{i}\right) \leq w(B)$ with the $w$-left Property $(A)$ of the basis we get

$$
\begin{aligned}
\left\|x+t 1_{\varepsilon A}\right\| & \leq \sum_{1}^{N}\left\|\frac{x}{N}+t 1_{\varepsilon A_{i}}\right\| \\
& \leq C_{l a} N\left\|\frac{x}{N}+t 1_{\eta B}\right\| \\
& \leq C_{l a}\left(N\left\|x+t 1_{\eta B}\right\|+(N-1)\|x\|\right) \\
& \leq C_{l a}\left(N\left\|x+t 1_{\eta B}\right\|+C_{l a}(N-1)\left\|x+t 1_{\eta B}\right\|\right) \\
& \leq \frac{4 C_{l a}^{2}}{\alpha}\left\|x+t 1_{\eta B}\right\|
\end{aligned}
$$

and this proves that the basis satisfies left Property $(A)$.

Let $\left(e_{n}\right)$ satisfies left Property $(A)$ with constant $C$. Choose $x \in X, t \geq \sup _{n}\left|e_{n}^{*}(x)\right|, \varepsilon, \eta \in$ $\mathcal{A}, A, B \in \mathbb{N}^{<\infty}$ with $A<B, w(A) \leq w(B)$ and $\operatorname{supp}(x) \cap(A \cup B)=\emptyset$. Then $|A| \leq \frac{1}{\alpha}|B|$ and we can partition $A$ into $N$ sets $A_{1}, \ldots, A_{N}$ with $\left|A_{i}\right| \leq|B|, A_{i}<B$ and $N \leq 1+\frac{1}{\alpha}$.

Now from the left Property $(A)$ of the basis we get

$$
\begin{aligned}
\left\|x+t 1_{\varepsilon A}\right\| & \leq \sum_{1}^{N}\left\|\frac{x}{N}+t 1_{\varepsilon A_{i}}\right\| \\
& \leq C N\left\|\frac{x}{N}+t 1_{\eta B}\right\| \\
& \leq C\left(N\left\|x+t 1_{\eta B}\right\|+(N-1)\|x\|\right) \\
& \leq C\left(N\left\|x+t 1_{\eta B}\right\|+C(N-1)\left\|x+t 1_{\eta B}\right\|\right) \\
& \leq 2 C^{2}\left(1+\frac{1}{\alpha}\right)\left\|x+t 1_{\eta B}\right\| .
\end{aligned}
$$

Thus $\left(e_{n}\right)$ satisfies $w$-left Property $(A)$.

Proposition 3.16. Let $w$ be a weight sequence with $0<\inf w_{n} \leq \sup w_{n}<\infty$. Then $\left(e_{n}\right)$ satisfies w-right Property $(A)$ if and only if $\left(e_{n}\right)$ satisfies right Property $(A)$.

Theorem 3.17. Let $w$ be a weight sequence with $0<\inf w_{n} \leq \sup w_{n}<\infty$. If a basis $\left(e_{n}\right)$ satisfies both w-left Property $(A)$ and w-right Property $(A)$ then the basis satisfies w-Property $(A)$.

Proof. Let $0<\alpha=\inf w_{n} \leq \sup w_{n}=1$ and $\left(e_{n}\right)$ satisfies both $w$-left Property $(A), w$-right Property $(A)$. Then form Propositions 3.15, 3.16 and Lemma 3.14, it follows that the basis satisfies Property $(A)$. Let $C$ be the constant for Property $(A)$. 
Let $x \in X, t \geq \sup _{j}\left|e_{j}^{*}(x)\right|, A, B \in \mathbb{N}^{<\infty}$ with $A \cap B=\emptyset, w(A) \leq w(B), \operatorname{supp}(x) \cap(A \cup B)=$ $\emptyset$ and $\varepsilon, \eta \in \mathcal{A}$. $w(A) \leq w(B)$ gives $|A| \leq \frac{1}{\alpha}|B|$ and thus we we can partition $A$ into $N$ sets $A_{1}, \ldots, A_{N}$ with $\left|A_{i}\right| \leq|B|, N \leq 1+\frac{1}{\alpha}$. Using the arguments similar to Proposition [3.15] we get $\left\|x+1_{\varepsilon A}\right\| \leq 2 C^{2}\left(1+\frac{1}{\alpha}\right)\left\|x+t 1_{\eta B}\right\|$ and this proves that the basis satisfies $w$-Property $(A)$.

Next, we will prove the similar result for a weight sequence $\left(w_{n}\right)$ with $\sum w_{n}=\infty$ and $\sup w_{n}<\infty$. We will consider some other conditions on the weight sequences in the next section for any basis satisfying weaker conditions than $w$-left Property $(A)$ and $w$-right Property (A).

Theorem 3.18. Let $w$ be a weight sequence with $\sum w_{n}=\infty$ and $\sup w_{n}<\infty$. If a basis $\left(e_{n}\right)$ satisfies both w-left Property $(A)$ and w-right Property $(A)$ then $\left(e_{n}\right)$ satisfies $w$-Property $(A)$.

Proof. Observe that from [2, Lemma 2.4] it follows that for the proof of this result it is sufficient to consider $x \in X$ with $\operatorname{supp}(x)<\infty$. Let $x \in X, t \geq \sup _{n}\left|e_{n}^{*}(x)\right|, \varepsilon, \eta, \gamma \in \mathcal{A}$, $A, B \in \mathbb{N}^{<\infty}$ with $w(A) \leq w(B), A \cap B=\emptyset$ and $\operatorname{supp}(x) \cap(A \cup B)=\emptyset$. Let $C$ be the constant for $w$-left Property $(A)$ and $w$-right Property $(A)$.

If $w(B) \leq \lim \sup _{n \rightarrow \infty} w_{n}$ then from Proposition 4.1 it follows that $\left\|1_{\varepsilon A}\right\|,\left\|1_{\eta B}\right\| \leq 4 C$. Thus we get

$$
\begin{aligned}
\left\|x+t 1_{\varepsilon A}\right\| & \leq\left\|x+t 1_{\eta B}\right\|+\left\|t 1_{\varepsilon A}\right\|+\left\|t 1_{\eta B}\right\| \\
& \leq\left\|x+t 1_{\eta B}\right\|+8 C t \\
& =\left\|x+t 1_{\eta B}\right\|+8 C\left\|t 1_{\eta b}\right\| \\
& \leq\left\|x+t 1_{\eta B}\right\|+16 C K_{b}\left\|x+t 1_{\eta B}\right\|
\end{aligned}
$$

where $K_{b}$ is the basis constant of $\left(e_{n}\right)$ and $b \in B$.

If $w(B)>\lim \sup _{n \rightarrow \infty} w_{n}$ then $\sum w_{n}=\infty$ implies that we can choose $E \in \mathbb{N}<\infty, n \in \mathbb{N}$ such that $F=\{n \cup E\}>(A \cup B), w(E) \leq w(B) \leq w(F)$ and $\operatorname{supp}(x) \cap F=\emptyset$. Observe that $w(A) \leq w(F)$ thus from the $w$-left Property $(A)$ and $w$-right Property $(A)$ of the basis we can write

$$
\begin{aligned}
\left\|x+t 1_{\varepsilon A}\right\| & \leq C\left\|x+t 1_{\gamma F}\right\| \\
& \leq C\left\{\left\|x+t 1_{\gamma E}\right\|+t\left\|e_{n}\right\|\right\} \\
& \leq C\left\{C\left\|x+t 1_{\eta B}\right\|+2 K_{b}\left\|x+t 1_{\eta B}\right\|\right\} \\
& =\left(C^{2}+2 C K_{b}\right)\left\|x+t 1_{\eta B}\right\| .
\end{aligned}
$$

\section{WEIGHT-CONSERVATIVE AND WEIGHT-REVERSE CONSERVATIVE BASES}

First, we will prove the following improvement of [2, Proposition 3.10]. The proof of [2, Proposition 3.10] itself suggests that we do not need the full power of $w$-superdemocracy of 
the basis (see [2, Definition 3.6]). The canonical basis of the space $c_{0}$, the space of all real sequences converging to 0 , is $\left(e_{n}\right)$ where $e_{n}$ has only one non zero entry at the $n$-th place and this entry has value equal to 1.

Proposition 4.1. Let $\left(e_{n}\right)$ be a basis of a Banach space $X$.

(a) If $\left(e_{n}\right)$ is w-conservative basis with constant $C$ and $A \in \mathbb{N}<\infty$ with $w(A) \leq \lim \sup _{n \rightarrow \infty} w_{n}$ then $\max _{\varepsilon \in \mathcal{A}}\left\|1_{\varepsilon A}\right\| \leq 4 C$.

(b) If $\sup w_{n}=\infty$ and $\left(e_{n}\right)$ is $w$-conservative basis then $\left(e_{n}\right)$ is equivalent to the canonical basis of $c_{0}$.

(c) If $\sum w_{n}<\infty$ and $\left(e_{n}\right)$ is $w$-reverse conservative basis then $\left(e_{n}\right)$ is equivalent to the canonical basis of $c_{0}$.

(d) If inf $w_{n}=0$ and $\left(e_{n}\right)$ w-reverse conservative basis then $\left(e_{n}\right)$ contains a subsequence which is equivalent to the canonical basis of $c_{0}$.

Proof. (a) For any $A \in \mathbb{N}<\infty$ with $w(A) \leq \limsup _{n \rightarrow \infty} w_{n}$, we can choose $n_{1}>n_{0}>A$ such that $w(A)<w_{n_{0}}+w_{n_{1}}$. For any $\varepsilon \in \mathcal{A}$ we can break $A$ into two parts $A^{+}$and $A^{-}$ where $\varepsilon_{i}=1$ for all $i \in A^{+}$and $\varepsilon_{i}=-1$ for all $i \in A^{-}$. Since $A^{+}, A^{-}<\left\{n_{0}, n_{1}\right\}$ and $w\left(A^{+}\right), w\left(A^{-}\right) \leq w_{n_{0}}+w_{n_{1}}$ we get $\left\|1_{\varepsilon A}\right\| \leq\left\|1_{A^{+}}\right\|+\left\|1_{A^{-}}\right\| \leq 2 C\left\|e_{n_{0}}+e_{n_{1}}\right\| \leq 4 C$.

(b) For any given $A \in \mathbb{N}<\infty$ we can find $n>A$ with $w(n)>w(A)$. Thus for any $\varepsilon \in \mathcal{A}$ we get $\left\|1_{\varepsilon A}\right\| \leq 2 C\left\|e_{n}\right\| \leq 2 C$.

(c) Choose $n \in \mathbb{N}$ such that $\sum_{n+1}^{\infty} w_{i}<w_{1}$. If $A \in \mathbb{N}<\infty$ with $A>n$ then $A>1$ and $w(A)<w_{1}$. Now from the $w$-reverse conservative property we can write $\left\|1_{\varepsilon A}\right\| \leq$ $2 C\left\|e_{1}\right\| \leq 2 C$ for all $\varepsilon \in \mathcal{A}$.

(d) Choose $\left(n_{i}\right)_{i}$ such that $\sum_{i} w_{n_{i}}<\infty$. From $(c)$ it follows that $\left(e_{n_{i}}\right)$ is equivalent to the canonical unit vector basis of $c_{0}$.

In [5] the authors proved that if $w \in c_{0}$ then any $w$-almost greedy basis is weakly null. We now prove the similar result for $w$-reverse conservative basis.

Corollary 4.2. If $w \in c_{0}$ then any $w$-reverse conservative basis is weakly null.

Proof. First observe that if $w \in c_{0}$ then any subsequence $\left(w_{n_{k}}\right)$ contains a further sequence $\left(w_{n_{k_{i}}}\right)$ such that $\sum_{i} w_{n_{k_{i}}}<\infty$. Also if $\left(e_{n}\right)$ is $w$-reverse conservative basis then $\left(e_{n_{k}}\right)$ is $w^{\prime}$-reverse conservative where $w^{\prime}=\left(w_{n_{k}}\right)$.

Let $w \in c_{0}$ and $\left(e_{n}\right)$ be a $w$-reverse conservative basis of $X$ which is not weakly null. Then there exists a $f \in X^{*}$ such that $f\left(x_{n}\right) \nrightarrow 0$. We can find a subsequence $\left(e_{n_{k}}\right)$ such that $\left|f\left(e_{n_{k}}\right)\right| \longrightarrow \delta>0$. This implies that $\left(e_{n_{k}}\right)$ doesn't contains any weakly null sequence and thus there is no further subsequence of $\left(e_{n_{k}}\right)$ which is equivalent to the canonical unit vector basis of $c_{0}$. Proposition 4.1(c) implies that this contradicts the $w$-reverse conservative property of $\left(e_{n}\right)$.

Proposition 4.3. Let $w \in c_{0}$ and $\left(e_{n}\right)$ is both $w$-reverse conservative and conservative. Then $\left(e_{n}\right)$ is equivalent to the canonical basis of $c_{0}$. 
Proof. Let $A \in \mathbb{N}^{<\infty}$ and $C_{1}, C_{2}$ be the constant for $w$-reverse conservative and conservative property respectively. Since $w \in c_{0}$, we can find a subsequence $\left(e_{n_{i}}\right)$ such that $\sum_{i} e_{n_{i}}<\infty$. Choose $N>A$ such that $\sum_{N+1}^{\infty} w_{n_{i}}<w_{1}$. Now we can find a finite set $D \subset\left\{n_{i}: i \geq N+1\right\}$ with $|D| \geq|A|$ and $w(D)<w_{1}$.

From the conservative and $w$-reverse conservative property we have $\left\|1_{A}\right\| \leq C_{2}\left\|1_{D}\right\| \leq$ $C_{1} C_{2}\left\|e_{1}\right\|=C_{1} C_{2}$.

Thus for any $A \in \mathbb{N}<\infty$ and $\varepsilon \in \mathcal{A}$ we have $\left\|1_{\varepsilon A}\right\| \leq 2 C_{1} C_{2}$ and this concludes the proof.

Similar arguments as in section 3 yields the following results.

Theorem 4.4. Let $w$ be a weight sequence with $\sum w_{n}=\infty$ and $\sup w_{n}<\infty$. If a basis $\left(e_{n}\right)$ is both $w$-conservative and $w$-reverse conservative then $\left(e_{n}\right)$ is $w$-democratic.

Proposition 4.5. Let $w$ be a weight sequence with $0<\inf w_{n} \leq \sup w_{n}<\infty$. Then basis $\left(e_{n}\right)$ of a Banach space $X$ is w-conservative (w-reverse conservative) if and only if $\left(e_{n}\right)$ is conservative (reverse conservative).

Theorem 4.6. Let $w$ be a weight sequence with $0<\inf w_{n} \leq \sup w_{n}<\infty$. If a basis $\left(e_{n}\right)$ is both $w$-conservative and $w$-reverse conservative then $\left(e_{n}\right)$ is $w$-democratic.

Proof. Let $\left(e_{n}\right)$ is both $w$-conservative, $w$-reverse conservative and $0<\alpha=\inf w_{n} \leq \sup w_{n}=$ 1. From Proposition 4.5 it follows that the basis is both conservative and reverse conservative. Now from [4, Lemma 2.8] it follows that the basis is democratic. Let $C$ be the constant of democracy.

Let $A, B \in \mathbb{N}^{<\infty}$ with $w(A) \leq w(B)$. Thus $|A| \leq \frac{1}{\alpha}|B|$ and we can write $A=\cup_{1}^{N} A_{i}$ where $\left|A_{i}\right| \leq|B|$ and $N \leq 1+\frac{1}{\alpha}$. Now from the democracy property of the basis we get

$$
\left\|1_{A}\right\| \leq \sum_{1}^{N}\left\|1_{A_{i}}\right\| \leq C N\left\|1_{B}\right\| \leq C\left(1+\frac{1}{\alpha}\right)\left\|1_{B}\right\|
$$

and this proves that the basis is $w$-democratic.

\section{ACKNOWLEDGEMEnTS}

The author is thankful to Gideon Schechtman for numerous useful discussions.

\section{REFERENCES}

[1] F. Albiac and P.Wojtaszczyk, Characterization of 1-greedy basis, J. Approx. Theory 138 (2006), no. 1, $65-86$.

[2] P.M. Berna, S.J. Dilworth, D. Kutzarova, T. Oikhberg and B. Wallis, The weighted Property $(A)$ and greedy algorithms, arXiv:1803.05052,

[3] S.J. Dilworth, N.J. Kalton, D. Kutzarova and V.N. Temlyakov, The thresholding greedy algorithm, greedy basis, and duality, Constr. Approx. 19 (2003), no. 4, 575-597.

[4] S.J. Dilworth and Divya Khurana, Characterizations of almost greedy and partially greedy bases, arXiv:1805.06778.

[5] S.J. Dilworth, D. Kutzarova, V.N. Temlyakov and B. Wallis, Weighted almost greedy bases, arXiv:1803.02932. 
[6] G. Kerkyacharian, D. Picard and V.N.Temlyakov, Some inequlities for the tensor product greedy bases and weight-greedy bases, East. J. Approx. 12 (2006), 103-118.

[7] S.V. Konyagin and V.N.Temlyakov, A remark on greedy approximation in Banach spaces, East. J. Approx. 5 (1999), 365-379.

[8] P. Wojtaszczyk, Greedy algorithm for general biorthogonal systems, J. Approx. Theory 107 (2000), 293314.

(Divya Khurana) Department of Mathematics, The Weizmann Institute of Science, Rehovot, ISRAEL, E-mail : divya.khurana@weizmann.ac.il, divyakhurana11@gmail.com 